\title{
Graphical Solutions of Fuzzy Models
}

\author{
S. Sindu Devi ${ }^{1}, \mathrm{~K}$ Ganesan ${ }^{2}$ \\ \{sindudes@ srmist.edu.in ${ }^{1}$, ganesan.k@ srmist.edu.in $\left.{ }^{2}\right\}$
}

${ }^{1}$ Department of Mathematics, Faculty of Engineering and Technology, SRM Institute of Science \& Technology, Ramapuram, Chennai - 89, ${ }^{2}$ Department of Mathematics, Faculty of Engineering and

Technology,SRM Institute of Science \& Technology, Kattankulathur, Chennai - 603203

\begin{abstract}
In the knowledge of dynamical system Fuzzy Initial Value Problems (FIVPs) have been vigorouslyinvestigatedover the modeling of numerousexhilaratingactual world problemwith all branches of engineering andscience. In this paper, numerical solution isassociated(1)gh and (2)gh differentiability. We propose a fuzzy versions of Picard's Method, solutionsinlinear fuzzy differential equations with first-order. The proposed technique is exemplified by a real-life sample and found to be in good agreement. The efficiency and accuracy of the suggestedtechnique is demonstrated in a series of graphical representations.
\end{abstract}

Keywords: Fuzzy Number, Generalized differentiability, Fuzzy initial value problem, Fuzzy Picard's method..

\section{Introduction}

Fuzzy Picard's method is an iterative technique for finding solutions of fuzzy difference equation through successive approximations. It is used for transforming fuzzy linear differential equation to integral equation. The solution of this method gives accurate results for fuzzy linear differential equations as it uses the same step size and degrees of precision in the integration procedure. The Picard's existence and uniqueness theorem is used for higher instruction fuzzy differential equation and also for systems of simultaneous fuzzy differential equation. The fuzzy Picard's process resembles that of Fuzzy Taylor's expansion. The nth approximation is added to integral equation and is used for computation of $(n+1)$ th element of the sequence.

SadighBehzadi .Sh [1]and others received the Picard technique for addressing the thePainlevé I and quadratic Riccaticonditions below fluffy climate and summed up $\mathrm{H}$ differentiability. SinduDevi.S and K Ganesan [2] suggested iterative plan arrangement of additional request fluffy differential conditions fluffy beginning condition utilizing fluffy picard strategy under summed up $\mathrm{H}$ - differentiability. Ettoussi, S et.al[3] has examined to discover the force arrangement of an intuitionistic fluffy introductory worth issues by utilizing progressive estimate strategy and we demonstrate that the rough arrangement combine consistently in t to the specific arrangement. Hussein ALKasasbeh et.al [4] have proposed new guess techniques for tackling frameworks of common differential conditions (SODEs) by fluffy change (FzT). A class of Volterraintegro-differential conditions, has been stretched out to tackle issues including Caputo fluffy fragmentary differential conditions Picard-like [numerical conspire by, Jorge E. Macias-Diaz[5].and StefaniaTomasiello 
In this article, we introduce first order fuzzy differentials equation under (i) - gh and (ii) gh differentiability using fuzzy Picard's techniquebeingwithindividuality solutions set satisfies the given system with a certain possibility. It shown that at anytime the solution constitutes a fuzzy region and alpha-cuts in the graphical representation.

\section{Preliminaries}

This segment contains some elementarydefinition which is very useful throughout this paper.

Definition 2.1. "Fuzzy set $\tilde{a}$ clearreal number $R$ supposed fuzzy number association functions $\tilde{a}: R \rightarrow(0,1)$ succeeding:

i. $\quad \tilde{a}$ are convex, i.e, $\tilde{a}\left\{\left(\lambda x_{1-0}+(1-\lambda)\right) x_{2} \geq \min \left\{\left(\tilde{a}\left(x_{1}\right), \bar{a}\left(x_{2}\right)\right)\right\}\right.$, for all $x_{1}, x_{2} \in$ $R \& \lambda \in[1,0]$

ii. a areregular i.e., occurs an $x \in R$ such $\tilde{a}(x)=1-0$

iii. ãare piece-wise continuou

Definition 2.2. "A triangular fuzzynumber is denoted as $\tilde{a}=\left(a_{1}, a_{2}, a_{3}\right)$ andis definite by the membership function

$$
\tilde{a}=\left\{\begin{array}{cc}
0, & x \leq q, \\
\frac{x-a_{1-0}}{a_{2}-a_{1}}, & a_{1} \leq x \leq a_{2}, \\
\frac{a_{3}-x}{a_{3}-a_{2}}, & a_{2} \leq x \leq a_{3}, \\
0,0 & x \geq a_{3} .
\end{array}\right.
$$

\subsection{Parametric Representation offuzzy numbers.}

A fuzzy number $\tilde{a} \in F(R)$ also signifiedduo $\tilde{a}=(\underline{a}, \bar{a})$ of function $\underline{a}(\alpha)$ and $\bar{a}(\alpha)$ for $0 \leq \alpha \leq 1$ contents the subsequent requirement:

i. $\quad \underline{a}(\alpha)$ is a circumscribed monotonic cumulativeveraciousincessant functions.

ii. $\quad \bar{a}(\alpha)$ is a circumscribed monotonic lesseningleftwardincessant functions.

iii. $\quad \underline{a}(\alpha) \leq \bar{a}(\alpha), 0 \leq \alpha \leq 1$.

\section{Fuzzy Derivative}

Definition 3.1. [Hukugara Derivative] Deliberate a fuzzy mapping $F:(a, b) \rightarrow R$ and $t_{0} \in$ $(a, b) . F$ is differentiable $t_{0} \in(a, b)$ if existelement $F^{\prime}\left(t_{0}\right) R h>0$ adequatelyslight $\exists F\left(t_{0}+\right.$ h) $\left.\ominus F\left(t_{0}\right), F\left(t_{0}\right) \ominus F\left(t_{0}-h\right) \operatorname{limit} D\right)$

$$
\lim _{h \rightarrow 0_{+}} \frac{F\left(t_{0}+h\right) \ominus F\left(t_{0}\right)}{h}=\lim _{h \rightarrow 0_{-}} \frac{F\left(t_{0}\right) \ominus F\left(t_{0}-h\right)}{h}
$$

exists and are equal to $F^{\prime}\left(t_{0}\right)$.

\section{Fuzzy Initial Value Problem}

initial value problem is a systems of usual differential equation together with the first conditions. Consider a function of $n^{\text {th }}$ fuzzy differential equations initial complaint are

$$
\begin{gathered}
\tilde{y}^{n}(t)=\tilde{f}\left(t, y(t), y^{\prime}(t), \ldots, y^{n-1}(t)\right. \\
\tilde{y}\left(t_{0}\right)=y_{0}, \ldots, \tilde{y}^{n-1}\left(t_{0}\right)=y_{0} .
\end{gathered}
$$

By using Extension principle, the membership functions are 


$$
[\tilde{f}(t, \tilde{y})]^{\alpha}=\tilde{f}\left(t,[\tilde{y}]^{\alpha}\right)=\tilde{f}\left(t,\left[\underline{y}_{\alpha}, \tilde{y}_{\alpha}\right]\right)=\left(\min \tilde{f}\left(t,\left[\underline{y}_{\alpha}, \tilde{y}_{\alpha}\right]\right), \max \tilde{f}\left(t,\left[\underline{y}_{\alpha}, \tilde{y}_{\alpha}\right]\right)\right)
$$

\section{Analysis of the method:}

In this segment, we exemplify the idea of the method. Let us deliberate the subsequentoveralldifference equations.

$$
y^{\prime}(t)=u(t, y) . \quad \tilde{y}\left(t_{0}\right)=y_{0}
$$

Alteration of variables to alter the original condition to the origin.Openly, describe $\mathrm{w}=\mathrm{y}-\mathrm{y}_{0}$ and $\mathrm{x}=\mathrm{t}-\mathrm{t}_{0}$. With a new $\mathrm{f}$, the differential equations is

$$
\begin{gathered}
y^{\prime}(t)=u(t, y) . \begin{array}{c}
\tilde{y}(0) \\
\end{array} \quad 0 \\
\mathrm{y}_{\mathrm{n}+1}(\mathrm{t})=\mathrm{y}_{\mathrm{n}}(\mathrm{s})+\int_{0}^{t}(f(s, y(s)) d s
\end{gathered}
$$

werelate the picardtechnique for (i) $-\mathrm{gH}$ differentiability

$$
\left\{\begin{array}{l}
u_{n+1}(t, \alpha)=u_{n}(s, \alpha)+\int_{0}^{t} f\left(s, u_{n}(s, \alpha)\right) d s, \\
u_{n+1}(t, \alpha)=u_{n}(s, \alpha)+\int_{0}^{t} f\left(s, u_{n}(s, \alpha)\right) d s .
\end{array}\right.
$$

werelate the picardtechnique for (i) - $\mathrm{gH}$ differentiability

$$
\left\{\begin{array}{l}
u_{n+1}(t, \alpha)=u_{n}(s, \alpha)-\int_{0}^{t} f\left(s, u_{n}(s, \alpha)\right) d s, \\
u_{n+1}(t, \alpha)=u_{n}(s, \alpha)-\int_{0}^{t} f\left(s, u_{n}(s, \alpha)\right) d s .
\end{array}\right.
$$

Example 1: Solve $y^{\prime}=-y+t+1$. with initial conditionsy $(0)=(0.96,1,1.01)$ then find the solution at $\mathrm{t}=[0,1]$.

$$
\begin{aligned}
& \text { Use (i) - ghdifferentiabily }\left\{\begin{array}{l}
u_{n+1}(t, \alpha)=u_{n}(s, \alpha)+\int_{0}^{t} f\left(s, u_{n}(s, \alpha)\right) d s, \\
u_{n+1}(t, \alpha)=u_{n}(s, \alpha)+\int_{0}^{t} f\left(s, u_{n}(s, \alpha)\right) d s .
\end{array}\right. \\
& \text { and (ii) - gh differentiability }\left\{\begin{array}{l}
u_{n+1}(t, \alpha)=u_{n}(s, \alpha)-\int_{0}^{t} f\left(s, u_{n}(s, \alpha)\right) d s, \\
u_{n+1}(t, \alpha)=u_{n}(s, \alpha)-\int_{0}^{t} f\left(s, u_{n}(s, \alpha)\right) d s .
\end{array}\right.
\end{aligned}
$$




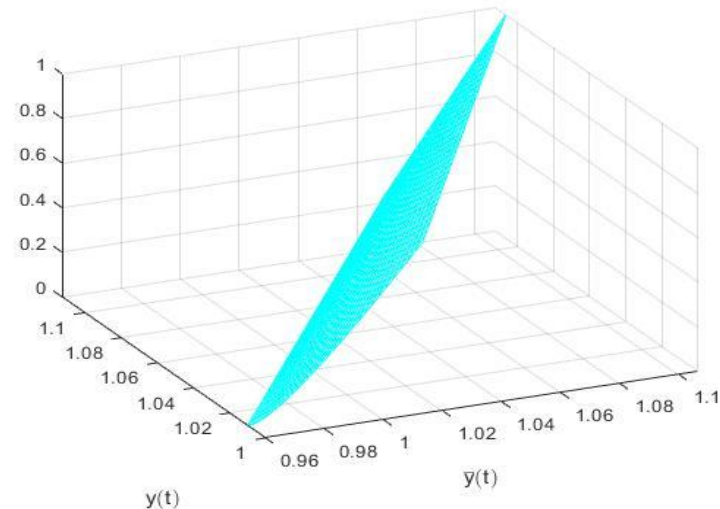

Figure 1: Approximate solution of fuzzy laplace transform under (i) $-\mathrm{gH}$ differentiability.

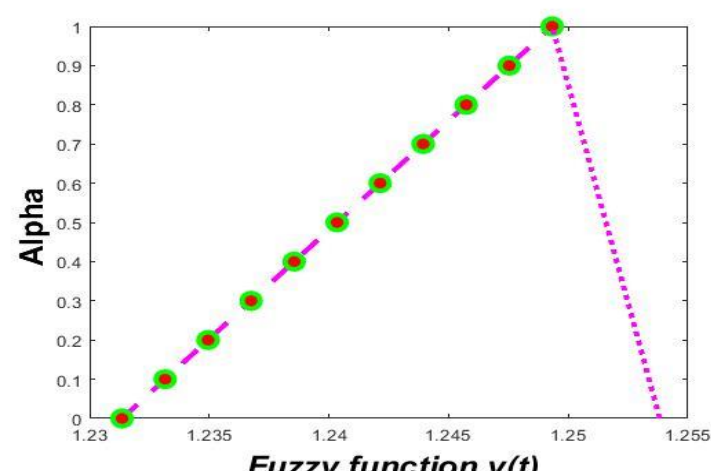

Figure 2: Graphical demonstration of fuzzy solution for $\mathrm{t}=0.8$ under (i) - gh differentiability.

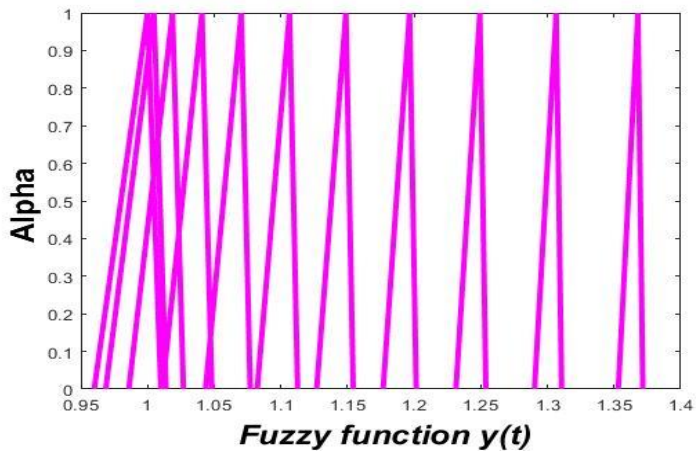

Figure 3: Graphical demonstrationof fuzzy solution for $\mathrm{t}=0,0.1,0.2$, , 1at fifth approximation under (i) - gh differentiability. 


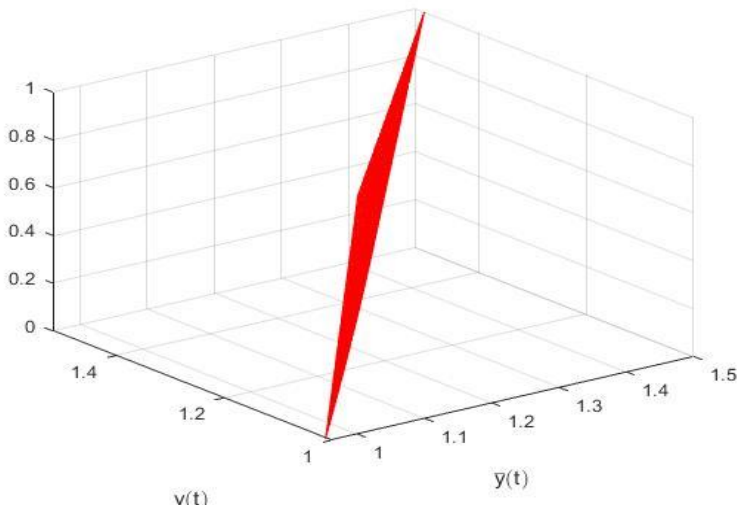

Figure 4: Approximate solution of fuzzy laplace transform under (ii) - $\mathrm{gH}$ differentiability

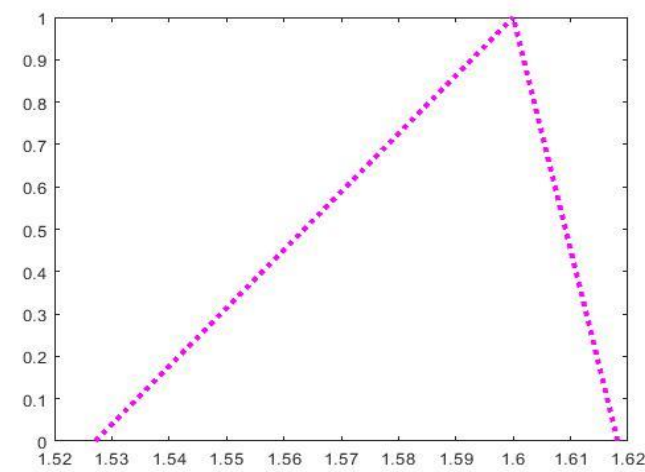

Figure 5: Graphical demonstration of fuzzy solution for $\mathrm{t}=0.8 \mathrm{under}$ (ii) -gh differentiability

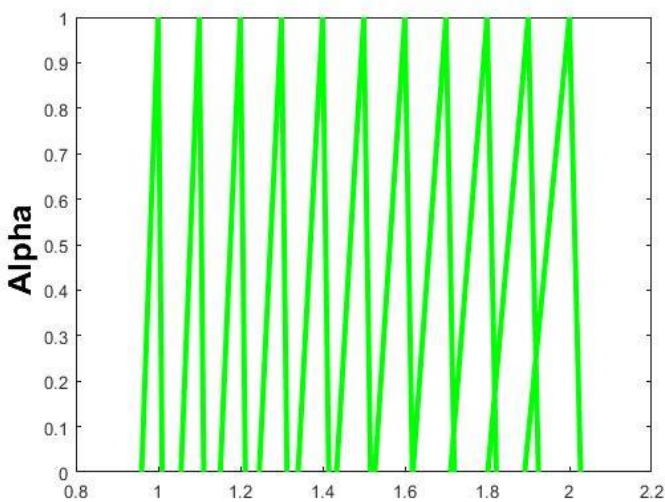

Fuzzy function $y(t)$

Figure 6: Graphical demonstration of fuzzy solution for $\mathrm{t}=0,0.1,0.2$, differentiability. 1 under (i) - gh 
Example 2: A tank at first contains 300 ladies of saline solution disintegrated in $\mathrm{c} \mathrm{lbs}$ of salts. Approaching the cisternat 3 ladies/min is brackish water with focus $\mathrm{k}$ lbs salts very much mixed combination greeneries rate 3 ladies/min. Let $\mathrm{y}(\mathrm{x})$ lbs be the salt in tanks at any time $\mathrm{t} \geq$ 0 . At that point $d y(x) / d x+(1 / 100) y(x)=k, x \varepsilon[0,1]$ with $y(0)=c$, if underlying disorder is existence displayed as fluffy number $\mathrm{c}=(1,2,3)$ and $\mathrm{k}=(1,2,4)$. Discover arrangement $\mathrm{x}=0.4$.

The below figures shows the approximate solutions of (i)-gh and (ii)-gh differentiability

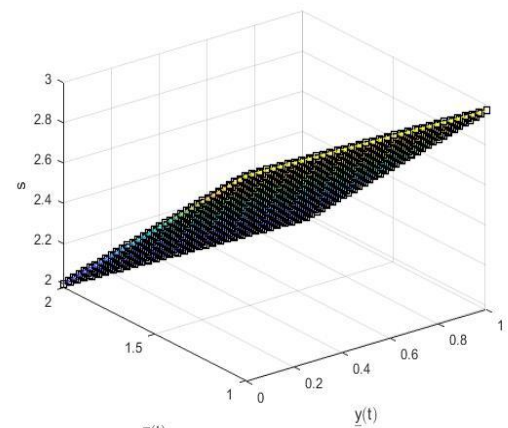

Figure 7: Approximate Solution of Fuzzy Picard's method under (i) -gh differentiability.

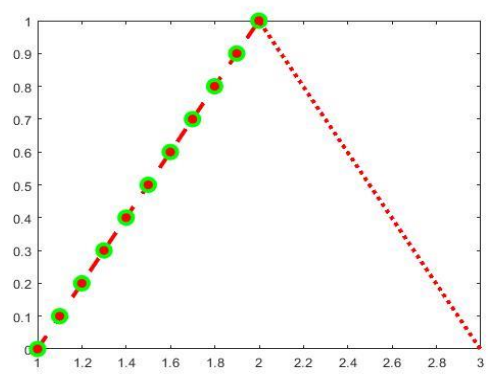

Figure 8: Graphical demonstration of fuzzy solution for $\mathrm{t}=0.1$.under (i) - gh differentiability.

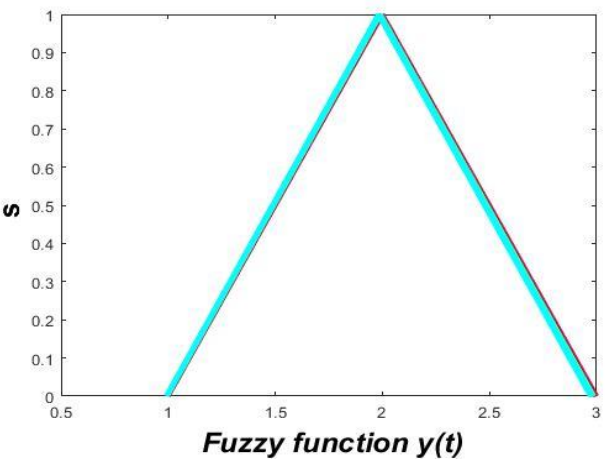

Figure 9: Graphical demonstration of fuzzy solution for different values $t=0,0.1,0.2$ approximation under(i)-gh differentiability 


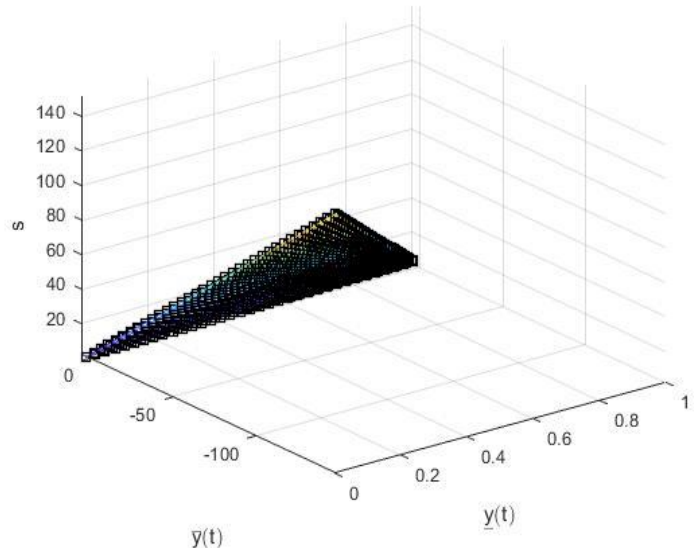

Figure 10: Approximate Solution of Fuzzy Picard's method under (ii) - gH differentiability.

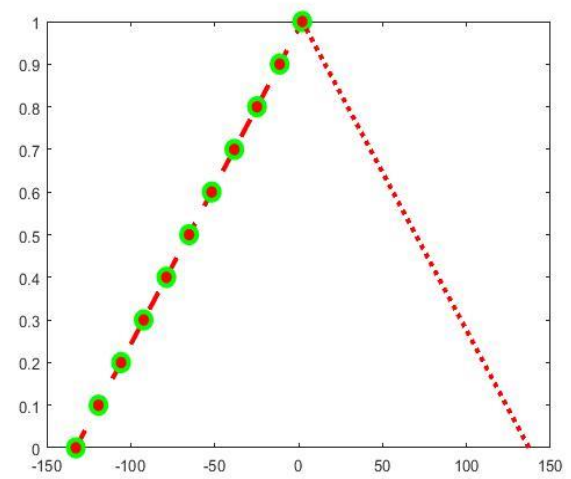

Figure 11: Graphical demonstration of fuzzy solution for $\mathrm{t}=0.1$ under (ii) - gh differentiability

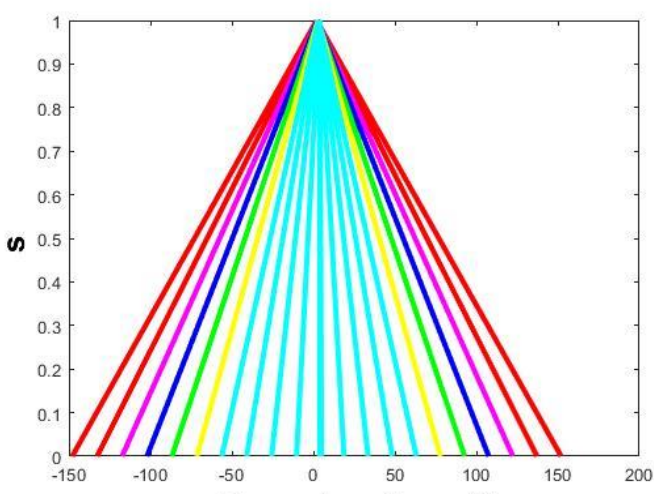

Fuzzy function $y(t)$

Figure 12: Graphical representation of fuzzy solution for dissimilar values $t=0,0.1,0.2$ differentiability. 


\section{Conclusions}

In this article, we have discussed Picard's technique for first order fuzzy differential equation proved under (i) - gh and (ii) - gh differentiability. For the efficacy of the suggested technique demonstrated by generous example, in the upcoming investigation relate the Picard technique to resolve a great class of FDEs.

\section{References}

[1] Sh. SadighBehzadi a , , BehnamVahdani b , T. Allahviranloo c , S. Abbasbandy, Application of fuzzy Picard method for solving fuzzy quadratic Riccati and fuzzy Painlevé I equations , Applied Mathematical Modelling 40 (2016) 8125-8137.

[2] S. Sindu Devi and K Ganesan, Fuzzy Picard's method for derivatives of second order, IOP Publishing IOP Conf. Series: Journal of Physics: Conf. Series 1000 (2018) 012038 doi :10.1088/1742-6596/1000/1/012038.

[3] R. Ettoussi, S. Melliani and L. S. Chadli, Approximate solution of intuitionistic fuzzy differential equations by using Picard's method, . Notes on Intuitionistic Fuzzy Sets 3 Vol. 24(2), 2018, 52-62 DOI: 10.7546/nifs.2018.24.2.52-62.

[4] Hussein ALKasasbeh , Irina Perfilieva D , Muhammad Zaini Ahmad 1 and ZainorRidzuanYahya New Approximation Methods Based on Fuzzy Transform for Solving SODEs:Applied System Innovation, , 1, 29(2018); doi:10.3390/asi1030029.

[5] StefaniaTomasiello and Jorge E. Macias-Diaz, Note on a Picard-like Method for Caputo Fuzzy Fractional Differential Equations, Appl. Math. Inf. Sci. 11, No. 1, 281-287 (2017). 